\title{
Positive Solutions for Elliptic Problems with the Nonlinearity Containing Singularity and Hardy-Sobolev Exponents
}

\author{
Yong-Yi Lan $(\mathbb{D}$, Xian Hu, and Bi-Yun Tang \\ School of Science, Jimei University, Xiamen 361021, China \\ Correspondence should be addressed to Yong-Yi Lan; lanyongyi@jmu.edu.cn
}

Received 4 February 2020; Accepted 16 April 2020; Published 27 April 2020

Guest Editor: Lishan Liu

Copyright (c) 2020 Yong-Yi Lan et al. This is an open access article distributed under the Creative Commons Attribution License, which permits unrestricted use, distribution, and reproduction in any medium, provided the original work is properly cited.

In this paper, we study multiplicity of positive solutions for a class of semilinear elliptic equations with the nonlinearity containing singularity and Hardy-Sobolev exponents. Using variational methods, we establish the existence and multiplicity of positive solutions for the problem.

\section{Introduction and Main Results}

Consider the following semilinear elliptic equations with Dirichlet boundary value conditions:

$$
\left(\begin{array}{ll}
-\Delta u-\mu \frac{u}{|x|^{2}}=\frac{|u|^{2^{*}(s)-2}}{|x|^{s}} u+\lambda u^{-\gamma}, & \text { in } \Omega, \\
u>0, & \text { in } \Omega, \\
u=0, & \text { in } \partial \Omega,
\end{array}\right.
$$

where $\Omega$ is a smooth bounded domain in $\mathbb{R}^{N}(N \geq 3), 0<$ $s<2,2^{*}(s)=2(N-s) / N-2$ is the Hardy-Sobolev critical exponent, $2^{*}=2^{*}(0)=2 N /(N-2)$ is the Sobolev critical exponent, $\mu<\bar{\mu}={ }^{\Delta}(N-2)^{2} / 4$, and $\gamma \in(0,1)$.

The energy functional associated with problem (1) is defined by

$$
\begin{aligned}
I_{\lambda}(u)= & \frac{1}{2} \int_{\Omega}\left(|\nabla u|^{2}-\mu \frac{u^{2}}{|x|^{2}}\right) d x-\frac{1}{2^{*}(s)} \int_{\Omega} \frac{|u|^{2^{*}(s)}}{|x|^{s}} d x \\
& -\frac{\lambda}{1-\gamma} \int_{\Omega}|u|^{1-\gamma} d x,
\end{aligned}
$$

for any $u \in H_{0}^{1}(\Omega)$. In general, a function $u$ is called a weak solution of problem (1) if $u \in H_{0}^{1}(\Omega)$ and $u(x)>0$ for all $x$ $\in \Omega$; it holds

$$
\begin{gathered}
\int_{\Omega}\left(\nabla u \cdot \nabla v-\mu \frac{u v}{|x|^{2}}-\frac{|u|^{2^{*}(s)-1}}{|x|^{s}} v-\lambda|u|^{-\gamma} v\right) d x \\
=0, \quad \forall v \in H_{0}^{1}(\Omega) .
\end{gathered}
$$

The paper by Crandall et al. [1] is the starting point on semilinear problem with singular nonlinearity. There is a large literature on singular nonlinearities (see [2-14] and references therein). In particular, the following Dirichlet problem

$$
\left(\begin{array}{l}
-\Delta u=u^{\beta}+\mu u^{-\gamma}, \quad \text { in } \Omega, \\
u>0, \quad \text { in } \Omega, \\
u=0, \quad \text { in } \partial \Omega,
\end{array}\right.
$$

has been shown in [2], in which the authors proved that problem (4) possesses at least one solution for $\mu>0$ small enough, and there exists no solution when $\mu$ is large. Chabrowski in [15] considered the Neumann boundary problems with singular superlinear nonlinearities by approximation and variational methods. When the superlinear term is subcritical, he obtained two solutions, a mountain-pass solution and a local minimizer solution. And, in the critical case, he obtained a local minimizer solution and proved that there is no moutain-pass solution.

In recent years, people have paid much attention to the existence of solutions for problems with the Sobolev critical 
exponent (the case that $s=0$ ) (see [16-21] and the references therein); some authors also considered the singular problems with the Hardy-Sobolev critical exponent (the case that $s \neq 0$ ) (see [22-27] and the references therein).

Up to our knowledge, the literature does not contain any result on the existence of positive solutions to the problem (1) with the nonlinearity containing singularity and HardySobolev exponents. Motivated by reasons above, the aim of this paper is to show the existence of positive solutions of problem (1). We study problem (1) and obtain at least two solutions via the Nehari method. It is well-known that the singular term leads to the nondifferentiability of the functional $I$ on $H_{0}^{1}(\Omega)$, so $I$ does not belong to $C^{1}\left(H_{0}^{1}(\Omega), \mathbb{R}\right)$. In order to get the existence of multiple positive solutions of problem (1), we use the Nehari method and differentiate the two solutions by their different Nehari-type sets.

The main result can be described as follows.

Theorem 1. Let $0<s<2, \mu<\bar{\mu}={ }^{\Delta}(N-2)^{2} / 4$ and $\gamma \in(0,1)$. Then, there exists $\lambda^{*}>0$ small enough, such that problem (1) has at least two positive solutions for any $\lambda \in\left(0, \lambda^{*}\right)$.

The paper is organized as follows: in Section 2, we give some preliminaries; in Section 3, we prove Theorem 1. This idea is essentially introduced in 20]. Throughout this paper, we make use of the following notations:

(i) The norm in $H_{0}^{1}(\Omega)$ is denoted by

$$
\|u\|^{2}=\int_{\Omega}\left(|\nabla u|^{2}-\mu \frac{u^{2}}{|x|^{2}}\right) d x
$$

By Hardy inequality [28], we easily derive that the norm is equivalent to the usual norm:

$$
\|u\|_{0}^{2}=\int_{\Omega}|\nabla u|^{2} d x
$$

(ii) $D^{1,2}\left(\mathbb{R}^{N}\right)(N \geq 3)$ denotes the space of the functions $u \in L^{2^{*}}\left(\mathbb{R}^{N}\right)$ such that $|\nabla u| \in L^{2}\left(\mathbb{R}^{N}\right)$, endowed with scalar product and norm, respectively, given by

$$
\begin{aligned}
\langle u, v\rangle_{\mathbb{R}^{N}} & =\int_{\mathbb{R}^{N}}\left(\nabla u \cdot \nabla v-\mu \frac{u v}{|x|^{2}}\right) d x, \\
\|u\|_{\mathbb{R}^{N}}^{2} & =\int_{\mathbb{R}^{N}}\left(|\nabla u|^{2}-\mu \frac{u^{2}}{|x|^{2}}\right) d x
\end{aligned}
$$

that coincides with the completion of $C_{0}^{\infty}\left(\mathbb{R}^{N}\right)$ with respect to the $L^{2}$-norm of the gradient. By Hardy inequality [28], we easily derive that the norm is equivalent to the usual norm:

$$
\|u\|_{0, \mathbb{R}^{N}}^{2}=\int_{\mathbb{R}^{N}}|\nabla u|^{2} d x
$$

in $D^{1,2}\left(\mathbb{R}^{N}\right)$.

(iii) The norm in $L^{p}(\Omega)$ is denoted by $|\cdot|_{p}$

(iv) $C, C_{0}, C_{1}, C_{2}, \cdots$ denote positive constants

\section{Preliminaries}

In this section, we will study the unperturbed problem

$$
\begin{cases}-\Delta u-\mu \frac{u}{|x|^{2}}=\frac{|u|^{2^{*}(s)-2}}{|x|^{s}} u, & x \in \mathbb{R}^{N}, \\ u \in D_{r}^{1,2}\left(\mathbb{R}^{N}\right), u>0, & x \in \mathbb{R}^{N} .\end{cases}
$$

It is well-known that the nontrivial solutions of problem (9) are equivalent to the nonzero critical points of the energy functional

$$
\begin{aligned}
I_{0}(u)= & \frac{1}{2} \int_{\mathbb{R}^{N}}\left(|\nabla u|^{2}-\mu \frac{u^{2}}{|x|^{2}}\right) d x \\
& -\frac{1}{2^{*}(s)} \int_{\mathbb{R}^{N}} \frac{\left|u^{+}\right|^{2^{*}(s)}}{|x|^{s}} d x, \quad u \in D_{r}^{1,2}\left(\mathbb{R}^{N}\right) .
\end{aligned}
$$

Obviously, the energy functional $I_{0}(u)$ is well-defined and is of $C^{2}$ with derivatives given by

$$
\begin{aligned}
&\left\langle I_{0}^{\prime}(u), v\right\rangle= \int_{\mathbb{R}^{N}}\left(\nabla u \cdot \nabla v-\mu \frac{u v}{|x|^{2}}\right) d x \\
&-\int_{\mathbb{R}^{N}} \frac{\left|u^{+}\right|^{2^{*}(s)-1}}{|x|^{s}} v d x, \quad u, v \in D_{r}^{1,2}\left(\mathbb{R}^{N}\right), \\
&\left\langle I_{0}^{\prime \prime}(u) v, w\right\rangle= \int_{\mathbb{R}^{N}}\left(\nabla v \cdot \nabla w-\mu \frac{v w}{|x|^{2}}\right) d x \\
&-\int_{\mathbb{R}^{N}} \frac{\left(2^{*}(s)-1\right)\left|u^{+}\right|^{2^{*}(s)-2}}{|x|^{s}} v w d x, \\
& u, v, w \in D_{r}^{1,2}\left(\mathbb{R}^{N}\right) .
\end{aligned}
$$

For all $\varepsilon>0$, it is well-known that the function

$$
\begin{aligned}
z_{\varepsilon}(x)= & \left(\frac{2 \varepsilon^{(2-s) \sqrt{\mu-\mu} / \sqrt{\mu}}(N-s)(\bar{\mu}-\mu)}{\sqrt{\bar{\mu}}}\right)^{\sqrt{\mu} / 2-s} / \\
& \cdot\left(| x | ^ { \sqrt { \mu } - \sqrt { \mu - \mu } } \left(\varepsilon^{(2-s) \sqrt{\mu-\mu} / \sqrt{\mu}}+|x|^{(2-s) \sqrt{\mu-\mu} / \sqrt{\mu}}\right.\right.
\end{aligned}
$$

solves equation (9) and satisfies

$$
\int_{\mathbb{R}^{N}}\left(\left|\nabla z_{\varepsilon}\right|^{2}-\mu \frac{z_{\varepsilon}^{2}}{|x|^{2}}\right) d x=\int_{\mathbb{R}^{N}} \frac{\left|z_{\varepsilon}\right|^{2^{*}(s)}}{|x|^{s}} d x .
$$


Moreover $z_{\varepsilon}(x)$ is the extremal function of the minimization problem

$$
\begin{aligned}
& S:=\inf \left\{\frac{\int_{\mathbb{R}^{N}}\left(|\nabla u|^{2}-\mu\left(u^{2} /|x|^{2}\right)\right) d x}{\left.\left(\int_{\mathbb{R}^{N}}\left(|u|^{2 *(s)} /|x|^{s}\right) d x\right)^{2 / 2^{*}(s)}: u \in D^{1,2}\left(\mathbb{R}^{N}\right), \quad u \neq 0\right\},}\right. \\
& S^{N-s / 2-s}=\int_{\mathbb{R}^{N}}\left(\left|\nabla z_{\varepsilon}\right|^{2}-\mu\left(\left.z_{\varepsilon}^{2}|| x\right|^{2}\right)\right) d x=\int_{\mathbb{R}^{N}}\left|z_{\varepsilon}\right|^{2 *(s)} /|x|^{s} d x .
\end{aligned}
$$

In view of $[27,29]$, we have the following exact local behavior of the solutions of (1).

Lemma 2. Let $0<s<2, \mu<\bar{\mu}={ }^{\Delta}(N-2)^{2} / 4$. If $u \in H_{0}^{1}(\Omega)$ is a positive solution of (1), then there exists $r>0$ small enough and some positive constants $C_{1}$ and $C_{2}$ such that

$$
C_{1}|x|^{-(\sqrt{\mu}-\sqrt{\mu-\mu})} \leq|u(x)| \leq C_{2}|x|^{-(\sqrt{\mu}-\sqrt{\mu-\mu})}, \quad x \in B(0, r) \backslash\{0\} .
$$

Define $\zeta \in C_{0}^{1}(\Omega)$ such that $|\zeta(x)| \leq 1,|\nabla \zeta(x)| \leq c$ for any $x \in \Omega, \zeta(x) \geq 0$ for all $\in \Omega, 0 \in \Omega$, and

$$
\zeta(x)=\left\{\begin{array}{lll}
1, & \text { if } & |x| \leq \delta \\
0, & \text { if } & |x| \geq 2 \delta .
\end{array}\right.
$$

Denote $v_{\varepsilon}(x)=\zeta(x) z_{\varepsilon}(x)$. Then, using an argument similar to [30], we have the following lemma.

Lemma 3. Let $u \in H_{0}^{1}(\Omega)$ be a weak solution of problem (1). Then, for $\varepsilon>0$ small enough

$$
\begin{aligned}
\int_{\Omega} \frac{|u|^{2^{*}(s)-1}}{|x|^{s}} v_{\varepsilon} d x & =O\left(\varepsilon^{N-2 / 4-2 s}\right), \\
\int_{\Omega} u \frac{\left|v_{\varepsilon}\right|^{2^{*}(s)-1}}{|x|^{s}} d x & =O\left(\varepsilon^{N-2 / 4-2 s}\right), \\
\int_{\Omega}\left(\left|\nabla v_{\varepsilon}\right|^{2}-\mu \frac{v_{\varepsilon}^{2}}{|x|^{2}}\right) d x & =S^{N-s / 2-s}+O\left(\varepsilon^{N-s / 2-s}\right)+O\left(\varepsilon^{N-2 / 2-s}\right), \\
\int_{\Omega} \frac{\left|v_{\varepsilon}\right|^{2^{*}(s)}}{|x|^{s}} d x & \geq S^{N-s / 2-s}-O\left(\varepsilon^{N-2 / 2-s}\right), \\
\int_{\Omega}\left|v_{\varepsilon}\right|^{p} d x & =O\left(\varepsilon^{p(N-2) / 4-2 s}\right) .
\end{aligned}
$$

Next, we define some Nehari-type sets, which are relevant in getting multiple positive solutions. Denote

$$
\begin{aligned}
& \mathcal{N}_{\lambda}=\left\{u \in H_{0}^{1}(\Omega):\|u\|^{2}=\int_{\Omega}\left(\frac{|u|^{2^{*}(s)}}{|x|^{s}}+\lambda|u|^{1-\gamma}\right) d x, \quad u \neq 0\right\}, \\
& \mathcal{N}_{\lambda}^{+}=\left\{u \in \mathcal{N}_{\lambda}:(1+\gamma)\|u\|^{2}>\left(2^{*}(s)-1+\gamma\right) \int_{\Omega} \frac{|u|^{2^{*}(s)}}{|x|^{s}} d x\right\}, \\
& \mathcal{N}_{\lambda}^{0}=\left\{u \in \mathcal{N}_{\lambda}:(1+\gamma)\|u\|^{2}=\left(2^{*}(s)-1+\gamma\right) \int_{\Omega} \frac{|u|^{2^{*}(s)}}{|x|^{s}} d x\right\}, \\
& \mathcal{N}_{\lambda}^{-}=\left\{u \in \mathcal{N}_{\lambda}:(1+\gamma)\|u\|^{2}<\left(2^{*}(s)-1+\gamma\right) \int_{\Omega} \frac{|u|^{2^{*}(s)}}{|x|^{s}} d x\right\} .
\end{aligned}
$$

Define the minimization problems

$$
d_{+}(\lambda):=\inf _{u \in \mathcal{N}_{\lambda}^{+}} I_{\lambda}(u)
$$

Since

$$
\begin{aligned}
I_{\lambda}(u)= & \frac{1}{2} \int_{\Omega}\left(|\nabla u|^{2}-\mu \frac{u^{2}}{|x|^{2}}\right) d x-\frac{1}{2^{*}(s)} \int_{\Omega} \frac{|u|^{2^{*}(s)}}{|x|^{s}} d x \\
& -\frac{\lambda}{1-\gamma} \int_{\Omega}|u|^{1-\gamma} d x,
\end{aligned}
$$

it is easy to see that $d_{+}(\lambda)<0$ for $\lambda>0$ and $d_{+}(\lambda) \longrightarrow 0$ as $\lambda \longrightarrow 0$. Take $\lambda_{3}>0$ such that $d_{+}(\lambda)+N^{-1} S^{N-s / 2-s}>0$ for any $\lambda \in\left(0, \lambda_{3}\right)$. Denote

$$
\begin{aligned}
\lambda_{4}= & \frac{2^{*}(s)-2}{2^{*}(s)+\gamma-1} S^{(1+\gamma)(N-s / 4-2 s)+1-\gamma / 2} \\
& \cdot\left(\frac{1+\gamma}{2^{*}(s)-\gamma-1}\right)^{(1+\gamma)(N-2 / 4-2 s)}|\Omega|^{\left(1-2^{*}(s)-\gamma\right) / 2^{*}(s)},
\end{aligned}
$$

and set $\lambda^{*}=\min \left\{\lambda_{3}, \lambda_{4}\right\}$.

Lemma 4. If $\lambda \in\left(0, \lambda^{*}\right)$, then $\mathcal{N}_{\lambda}^{0}=\varnothing$. Moreover, for any $u \neq 0$, there exists a unique $t^{+}=t^{+}(u)>0$ such that $t^{+}(u) u$ $\in \mathcal{N}_{\lambda}^{-}$and

$$
\begin{aligned}
& t^{+}>T_{m}:=\left(\frac{\|u\|^{2}}{\left(2^{*}(s)-1\right) \int_{\Omega}\left(|u|^{2^{*}(s)} /|x|^{s}\right) d x}\right)^{1 /\left(2^{*}(s)-2\right)}, \\
& I_{\lambda}\left(t^{+} u\right)=\max _{t \geq T_{m}} I_{\lambda}(t u),
\end{aligned}
$$

and there exists a unique $t^{-}=t^{-}(u)>0$ such that $t^{-}(u) u$ $\in \mathcal{N}_{\lambda}^{+}$and

$$
t^{-}<T_{m} \quad \text { and } \quad I_{\lambda}\left(t^{-} u\right)=\inf _{0 \leq t \leq T_{m}} I_{\lambda}(t u) .
$$

Proof. The proof is similar to [30]. We omit the details. 


\section{Proof of Theorem 1}

In this section, we will prove Theorem 1 . The proof of Theorem 1 is based on solving the minimization problem (18) and the minimization problem

$$
d_{-}(\lambda):=\inf _{u \in \mathscr{N}^{-}} I_{\lambda}(u)
$$

We divide the proof into two steps. In the first step, we prove that if there is $w \in \mathcal{N}_{\lambda}^{+}$such that $d_{+}(\lambda)=I_{\lambda}(w)$ and there is $v \in \mathscr{N}_{\lambda}^{-}$such that $d_{-}(\lambda)=I_{\lambda}(v)$, then $w$ and $v$ are two positive weak solutions of (1). In the second step, we prove that the minima $d_{+}(\lambda)$ in (18) and $d_{-}(\lambda)$ in (23) are achieved, respectively.

Step 1. Let $w \in \mathcal{N}_{\lambda}^{+}$be such that $d_{+}(\lambda)=I_{\lambda}(w)$ and $v \in \mathcal{N}_{\lambda}^{-}$ such that $d_{-}(\lambda)=I_{\lambda}(v)$.

Lemma 5. For each $\varphi \in H_{0}^{1}(\Omega)$ and $\varphi \geq 0$, we have the following:

There is $\eta_{0}$ such that $I_{\lambda}(w+\eta \varphi) \geq I_{\lambda}(w)$ for each $0 \leq$ $\eta \leq \eta_{0}$

(ii) $t_{\eta}^{-} \longrightarrow 1$ as $\eta \longrightarrow 0^{+}$, where for each $\eta \geq 0$, $t_{\eta}^{-}$is the unique positive number satisfying $t_{\eta}^{-}(v+\eta \varphi) \in \mathcal{N}_{\lambda}^{-}$

Proof. The proof follows exactly the scheme in the proof of Lemma 3 in [31].

Lemma 6. For each $\varphi \in H_{0}^{1}(\Omega)$ and $\varphi \geq 0$, we have $w^{-\gamma} \varphi$ and $v^{-\gamma} \varphi \in L^{1}(\Omega)$. Moreover,

$$
\begin{gathered}
\int_{\Omega}\left(\nabla w \nabla \varphi-\mu \frac{w \varphi}{|x|^{2}}-\frac{|w|^{2^{*}(s)-1}}{|x|^{s}} \varphi-\lambda|w|^{-\gamma} \varphi\right) d x \geq 0, \\
\int_{\Omega}\left(\nabla v \nabla \varphi-\mu \frac{v \varphi}{|x|^{2}}-\frac{|v|^{2^{*}(s)-1}}{|x|^{s}} \varphi-\lambda|v|^{-\gamma} \varphi\right) d x \geq 0 .
\end{gathered}
$$

In particular, $w, v>0$ for all $x \in \Omega \backslash\{0\}$.

Proof. We only prove (24) since the proof of (25) is similar. Let $\varphi \geq 0$ and $\varepsilon>0$. By (i) of Lemma 5 and simple computations, we have that

$$
\begin{aligned}
& \frac{\lambda}{1-\gamma} \int_{\Omega} \frac{(w+\varepsilon \varphi)^{1-\gamma}-w^{1-\gamma}}{\varepsilon} \mathrm{d} x \\
& \quad \leq \frac{1}{2 \varepsilon}\left(\|w+\varepsilon \varphi\|^{2}-\|w\|^{2}\right) \\
& \quad-\frac{1}{2^{*}(s) \varepsilon} \int_{\Omega}\left(\frac{|w+\varepsilon \varphi|^{2^{*}(s)}}{|x|^{s}}-\frac{|w|^{2^{*}(s)}}{|x|^{s}}\right) \mathrm{d} x .
\end{aligned}
$$

Since the right hand side of the inequality has a finite limit value as $\varepsilon \longrightarrow 0$, by direct calculations, we conclude $\varepsilon^{-1}\left((w+\varepsilon \varphi)^{1-\gamma}-w^{1-\gamma}\right)$ increases monotonically as $\varepsilon \longrightarrow 0$ and

$$
\begin{aligned}
& \lim _{\varepsilon \rightarrow 0} \frac{\left((w+\varepsilon \varphi)^{1-\gamma}-w^{1-\gamma}\right)}{\varepsilon} \\
& \quad=\left\{\begin{array}{lll}
0, & \text { if } \varphi(x)=0, \\
(1-\gamma) w^{-\gamma} \varphi, & \text { if } \varphi(x)>0 & \text { and } w(x)>0, \\
\infty, & \text { if } \quad \varphi(x)>0 & \text { and } w(x)=0 .
\end{array}\right.
\end{aligned}
$$

The Fatou lemma yields $w^{-\gamma} \varphi \in L^{1}(\Omega)$ and we get (24).

Since $w, v \geq 0$ and $w, v \equiv 0$, by the strong maximum principle, it follows that

$$
w, v>0, \quad \forall x \in \Omega \backslash\{0\}
$$

Lemma 7. We have that $w$ and $v$ are positive weak solutions of (1).

Proof. For any $\varphi \in H_{0}^{1}(\Omega)$ and $\eta>0$, we define $\psi=(w+\eta \varphi)$ and $\psi^{+}=\max \{\psi, 0\}$. Then, $\psi^{+} \in H_{0}^{1}(\Omega)$. Since $w \in \mathcal{N}_{\lambda}$, we obtain from (24) that

$$
\begin{aligned}
0 \leq & \int_{\Omega}\left(\nabla w \nabla \psi^{+}-\mu \frac{w \psi^{+}}{|x|^{2}}-\frac{|w|^{2^{*}(s)-1}}{|x|^{s}} \psi^{+}-\lambda|w|^{-\gamma} \psi^{+}\right) \mathrm{d} x \\
= & \int_{\{x \in \Omega, w+\eta \varphi>0\}}\left(\nabla w \nabla \psi^{+}-\mu \frac{w \psi^{+}}{|x|^{2}}-\frac{|w|^{2^{*}(s)-1}}{|x|^{s}} \psi^{+}-\lambda|w|^{-\gamma} \psi^{+}\right) \mathrm{d} x \\
= & \int_{\Omega}\left(\nabla w \nabla \psi-\mu \frac{w \psi}{|x|^{2}}-\frac{|w|^{2^{*}(s)-1}}{|x|^{s}} \psi-\lambda|w|^{-\gamma} \psi\right) \mathrm{d} x \\
& -\int_{\{x \in \Omega, w+\eta \varphi \leq 0\}}\left(\nabla w \nabla \psi-\mu \frac{w \psi}{|x|^{2}}-\frac{|w|^{2^{*}(s)-1}}{|x|^{s}} \psi-\lambda|w|^{-\gamma} \psi\right) \mathrm{d} x \\
\leq & \int_{\Omega}\left(\nabla w \nabla \varphi-\mu \frac{w \varphi}{|x|^{2}}-\frac{|w|^{2^{*}(s)-1}}{|x|^{s}} \varphi-\lambda|w|^{-\gamma} \varphi\right) \mathrm{d} x \\
& -\eta \int_{\{x \in \Omega, w+\eta \varphi \leq 0\}}\left(\nabla w \nabla \varphi-\mu \frac{u \varphi}{|x|^{2}}\right) \mathrm{d} x .
\end{aligned}
$$

Dividing by $\eta$ and letting $\eta \longrightarrow 0$, by $w \geq 0, \forall x \in \Omega$, we have the measure of $\{x \in \Omega, w+\eta \varphi \leq 0\}$ which tends to 0 as $\eta \longrightarrow 0$, and we get that

$$
\int_{\{x \in \Omega, w+\eta \varphi \leq 0\}} \nabla w \nabla \varphi \mathrm{d} x \longrightarrow 0
$$

Therefore,

$$
\int_{\Omega}\left(\nabla w \nabla \varphi-\mu \frac{w \varphi}{|x|^{2}}-\frac{|w|^{2^{*}(s)-1}}{|x|^{s}} \varphi-\lambda|w|^{-\gamma} \varphi\right) \mathrm{d} x \geq 0
$$

Since $\varphi$ is arbitrary, we get that $w$ is a solution of (1). Similarly, we can prove that $v$ is also a solution of (1).

Step 2. The minima $d_{+}(\lambda)$ and $d_{-}(\lambda)$ are achieved. The proof is exactly the same as [32]. We omit the details here. 
We point out that $v_{\varepsilon}$ and the exact local behavior of $w$ (see Lemma 2.) play essential roles. From Lemma 2., we have $C_{1}|x|^{-(\sqrt{\mu}-\sqrt{\bar{\mu}-\mu})} \leq|w(x)| \leq C_{2}|x|^{-(\sqrt{\bar{\mu}}-\sqrt{\bar{\mu}-\mu})}, \quad x \in B(0, r) \backslash\{0\}$.

So there is $m>0$ such that $w(x) \geq m$ for $x \in \operatorname{supp} w \backslash\{0\}$.

Lemma 8. Under the assumptions of Theorem 1,

$$
d_{-}(\lambda)<I_{\lambda}(w)+\frac{2-s}{2(N-s)} S^{N-s / 2-s}
$$

Proof. First, using an argument similar to the proofs in [31], we have $\eta_{*}>0$ such that $w+\eta_{*} v_{\varepsilon} \in \mathcal{N}_{\lambda}^{-}$. It remains to be proven that

$$
\sup \left\{I_{\lambda}\left(w+\eta v_{\varepsilon}\right): \eta>0\right\}<I_{\lambda}(w)+\frac{2-s}{2(N-s)} S^{N-s / 2-s}
$$
that

Since $w$ is a solution, we obtain by direct computation

$$
\begin{aligned}
I_{\lambda}\left(w+\eta v_{\varepsilon}\right)-I_{\lambda}(w)= & \frac{\eta^{2}}{2}\left\|v_{\varepsilon}\right\|^{2}+\eta \int_{\Omega}\left(\nabla w \nabla v_{\varepsilon}-\mu \frac{w v_{\varepsilon}}{|x|^{2}}\right) \mathrm{d} x \\
& -\int_{\Omega}\left(\frac{\left|w+\eta v_{\varepsilon}\right|^{2^{*}(s)}-w^{2^{*}(s)}}{2^{*}(s)|x|^{s}}\right) d x \\
& -\lambda \int_{\Omega}\left(\frac{\left(w+\eta v_{\varepsilon}\right)^{1-\gamma}}{1-\gamma}-\frac{w^{1-\gamma}}{1-\gamma}\right) d x \\
= & \frac{\eta^{2}}{2}\left\|v_{\varepsilon}\right\|^{2}-\int_{\Omega}\left(\frac{\left|w+\eta v_{\varepsilon}\right|^{2^{*}(s)}-w^{2^{*}(s)}}{2^{*}(s)|x|^{s}}\right. \\
& \left.-\frac{|w|^{2^{*}(s)-1} \eta v_{\varepsilon}}{|x|^{s}}\right) d x-\lambda \int_{\Omega}\left(\frac{\left(w+\eta v_{\varepsilon}\right)^{1-\gamma}}{1-\gamma}\right. \\
& \left.-\frac{w^{1-\gamma}}{1-\gamma}-w^{-\gamma} \eta v_{\varepsilon}\right) d x .
\end{aligned}
$$

Note that the following inequality (see [31]) holds: there is $\alpha>0$ and $0<\delta<N-s / N-2$ such that $\lambda\left((r+t)^{1-\gamma}\right.$ / $\left.1-\gamma-r^{1-\gamma} / 1-\gamma-r^{-\gamma} t\right) \geq-\alpha t^{\delta}$ for each $\mathrm{r} \geq \mathrm{m}$ and $\mathrm{t} \geq 0$.

By direct calculations, we can get that

$$
\begin{aligned}
I_{\lambda}\left(w+\eta v_{\varepsilon}\right)-I_{\lambda}(w) \leq & \frac{\eta^{2}}{2}\left\|v_{\varepsilon}\right\|^{2}-\frac{\eta^{2^{*}(s)}}{2^{*}(s)} \int_{\Omega} \frac{\left|v_{\varepsilon}\right|^{2^{*}(s)}}{|x|^{s}} d x \\
& -\eta^{2^{*}(s)-1} \int_{\Omega} w \frac{\left|v_{\varepsilon}\right|^{2^{*}(s)-1}}{|x|^{s}} d x \\
& +\alpha \eta^{\delta} \int_{\Omega}\left|v_{\varepsilon}\right|^{\delta} d x
\end{aligned}
$$

So $I_{\lambda}\left(w+\eta v_{\varepsilon}\right)-I_{\lambda}(w) \longrightarrow 0$ as $\eta \longrightarrow 0$ and $I_{\lambda}(w+$ $\left.\eta v_{\varepsilon}\right)-I_{\lambda}(w) \longrightarrow-\infty$ as $\eta \longrightarrow+\infty$. Hence, we only consider the right-hand side of the above inequality in the case of $\eta \in\left[\eta_{0}, \eta_{1}\right]$ for some $0<\eta_{0}<\eta_{1}<+\infty$. Hence, we obtain from Lemma 3 that

$$
\begin{aligned}
\sup _{\eta>0} I_{\lambda}\left(w+\eta v_{\varepsilon}\right)-I_{\lambda}(w) \leq & \frac{2-s}{2(N-s)}\left\|v_{\varepsilon}\right\|^{22^{*}(s) / 2^{*}(s)-2} \\
& -\left(\int_{\Omega} \frac{\left|v_{\varepsilon}\right|^{2^{*}(s)}}{|x|^{s}} \mathrm{~d} x\right)^{-2 / 2^{*}(s)-2} \\
& -O\left(\varepsilon^{N-2 / 4-2 s}\right)+O\left(\varepsilon^{\delta(N-2 / 4-2 s)}\right) \\
= & \frac{2-s}{2(N-s)} S^{N-s / 2-s}-O\left(\varepsilon^{N-s / 4-2 s}\right) \\
& -O\left(\varepsilon^{N-2 / 4-2 s}\right)+O\left(\varepsilon^{\delta(N-2 / 4-2 s)}\right) \\
< & \frac{2-s}{2(N-s)} S^{N-s / 2-s},
\end{aligned}
$$$$
\text { for } \varepsilon>0 \text { sufficiently small. }
$$

The proof is complete.

Lemma 9. The minimum $d_{-}(\lambda)$ in (23) is achieved by $v \in \mathcal{N}_{\lambda}^{-}$ with $I_{\lambda}(v)=d_{-}(\lambda)$.

Proof. Let $\left\{v_{n}\right\}$ be any sequence in $\mathcal{N}_{\lambda}^{-}$such that $I_{\lambda}\left(v_{n}\right)$ $\longrightarrow d_{-}(\lambda)$. It is easy to see that $\left\{v_{n}\right\}$ is bounded in $H_{0}^{1}(\Omega)$. Then, there exists a $v \in H_{0}^{1}(\Omega)$ and a subsequence of $\left\{v_{n}\right\}$, still denoted by $\left\{v_{n}\right\}$ such that

$$
v_{n} \rightarrow v, \quad \text { in } \quad H_{0}^{1}(\Omega) .
$$

Set $u_{n}=v_{n}-v$ and assume that

$$
\begin{gathered}
\left\|u_{n}\right\|^{2} \longrightarrow a^{2}, \\
\int_{\Omega} \frac{\left|u_{n}\right|^{2^{*}(s)}}{|x|^{s}} d x \longrightarrow b^{2^{*}(s)} .
\end{gathered}
$$

Since $v_{n} \in \mathcal{N}_{\lambda}^{-}$, according to Bre'zis-Lieb's Lemma (see Lemma 1.32 in MW [33]) and the Sobolev embedding theorem, one gets

$$
a^{2}+\|v\|^{2}=\lambda \int_{\Omega}|v|^{1-\gamma} d x+b^{2^{*}(s)}+\int_{\Omega} \frac{|v|^{2^{*}(s)}}{|x|^{s}} d x .
$$

We claim that $v \neq 0$. Indeed, if $v=0$, then $a^{2}=b^{2^{*}} \geq$ $S^{N-s / 2-s} \neq 0$ (since for any $u \in \mathcal{N}_{\lambda}^{-},\|u\|$ is bounded away from zero) and this means that

$$
\begin{aligned}
d_{-}(\lambda) & =\lim _{n \longrightarrow \infty} I_{\lambda}\left(v_{n}\right)=I_{\lambda}(0)+\frac{1}{2} a^{2}-\frac{1}{2^{*}(s)} b^{2^{*}(s)} \\
& \geq \frac{2-s}{2(N-s)} S^{N-s / 2-s}
\end{aligned}
$$

which contradicts the previous lemma. 
From the assumption $\lambda \in\left(0, \lambda^{*}\right)$, by Lemma 4 , we have $0<t^{+}<T_{m}<t^{-}$such that $t^{+} v \in \mathcal{N}_{\lambda}^{+}$and $t^{-} v \in \mathcal{N}_{\lambda}^{-}$. For $t>0$, we define

$$
\begin{aligned}
& \delta(t)=\frac{1}{2} a^{2} t^{2}-\frac{1}{2^{*}(s)} b^{2^{*}(s)} t^{2^{*}(s)} \\
& g(t)=I_{\lambda}(t v)+\delta(t)
\end{aligned}
$$

Now, we consider the cases

$$
\text { (i) } t^{-}<1
$$

(ii) $t^{-} \geq 1$ and $b>0$

(iii) $t^{-} \geq 1$ and $b=0$

Case (i). From $t^{-}<1$,

$$
g^{\prime}\left(t^{-}\right)=\left\langle I\left(t^{-} v\right), v\right\rangle+\delta^{\prime}\left(t^{-}\right)=a^{2} t^{-}-b^{2^{*}(s)}\left(t^{-}\right)^{2^{*}(s)-1}
$$

Define $h(t) \doteq a^{2} t-b^{2^{*}(s)}(t)^{2^{*}(s)-1}$, then $h(t)$ is increasing as $t<T_{m} ; h(t)$ is decreasing as $t>T_{m}$, so $h(t)$ has maximum value $h\left(T_{m}\right)$. As $t^{-}>T_{m}$, we have $g^{\prime}\left(t^{-}\right) \geq h\left(T_{m}\right)>0 . g^{\prime}$ (1) $=0$ and $g^{\prime}\left(t^{-}\right)>0$, so we can see that $g$ is increasing on $\left[t^{-}, 1\right]$. Then, we have

$$
\begin{aligned}
d_{-}(\lambda) & =g(1)>g\left(t^{-}\right) \geq I_{\lambda}\left(t^{-} v\right)=I_{\lambda}(0)+\frac{\left(t^{-}\right)^{2}}{2}\left(a^{2}-b^{2^{*}(s)}\right) \\
& >I_{\lambda}\left(t^{-} v\right) \geq d_{-}(\lambda)
\end{aligned}
$$

which is a contradiction.

Case (ii). We set $t_{0}=\left(a^{2} / b^{2^{*}(s)}\right)^{N-2 / 2(2-s)}$. We know that $\delta$ attains the unique maximum at $t_{0}$ and $\delta\left(t_{0}\right) \geq(2-s / 2(N-$ $s)) S^{N-s / 2-s}$. Moreover, $\delta^{\prime}(t)>0$ for $0<t<t_{0}$ and $\delta^{\prime}(t)<0$ for $t>t_{0}$.

From the assumption on $\lambda \in\left(0, \lambda^{*}\right)$, we know $g(1) \geq$ $g\left(t_{0}\right)$. If $t_{0} \leq 1$, we have

$$
\begin{aligned}
d_{-}(\lambda) & =g(1) \geq g\left(t_{0}\right)=I_{\lambda}\left(t_{0} v\right)+\delta\left(t_{0}\right) \\
& \geq I_{\lambda}\left(t_{0} v\right)+\frac{2-s}{2(N-s)} S^{N-s / 2-s},
\end{aligned}
$$

which contradicts the previous lemma. Thus, we have $t_{0}>1$. By virtue of $g^{\prime}(t) \leq 0$ for $t \geq 1$, we obtain $(\partial / \partial t) I_{\lambda}(t v) \leq-\delta^{\prime}$ $(t) \leq 0$ for $1 \leq t \leq t_{0}$ and

$$
\begin{aligned}
d_{-}(\lambda) & =g(1)=I_{\lambda}(v)+\frac{1}{2} a^{2}-\frac{1}{2^{*}(s)} b^{2^{*}(s)} \\
& \geq I_{\lambda}(v)+\frac{2-s}{2(N-s)} S^{N-s / 2-s},
\end{aligned}
$$

which also contradicts the previous lemma.

Case (iii). If $a \neq 0$, then we obtain from the fact that $v_{n}$ $\in \mathcal{N}_{\lambda}^{-}$by some computations that $\left.(\partial / \partial t) I_{\lambda}(t v)\right|_{t=1}<0$ and $\left.\left(\partial^{2} / \partial^{2} t\right) I_{\lambda}(t v)\right|_{t=1}<0$, from the definition of $\mathcal{N}_{\lambda}^{-}$, which contradicts $t^{-} \geq 1$. So

$$
\begin{aligned}
& a=0, \\
& v_{n} \longrightarrow v \text { in } H_{0}^{1}(\Omega) .
\end{aligned}
$$

Hence, we have $v \in \mathcal{N}_{\lambda}^{-}$with $I_{\lambda}(v)=d_{-}(\lambda)$. The proof is complete.

\section{Data Availability}

The data used to support the findings of this study are included within the article.

\section{Conflicts of Interest}

The authors declare that they have no competing interests.

\section{Acknowledgments}

This paper is supported by the National Natural Science Foundation of China (11671331) and National Foundation Training Program of Jimei University (ZP2020057).

\section{References}

[1] M. G. Crandall, P. H. Rabinowitz, and L. Tartar, "On a Dirichlet problem with a singular nonlinearity," Communications in Partial Differential Equations, vol. 2, no. 2, pp. 193222, 1977.

[2] M. M. Coclite and G. Palmieri, "On a singular nonlinear Dirichlet problem," Communications in Partial Differential Equations, vol. 23, no. 7, pp. 1315-1327, 1989.

[3] A. C. Lazer and P. J. Mckenna, "On a singular nonlinear elliptic boundary value problem," Proceedings of the American Mathematical Society, vol. 111, no. 3, pp. 721-730, 1991.

[4] Z. J. Zhang, "On a Dirichlet problem with a singular nonlinearity," Journal of Mathematical Analysis and Applications, vol. 194, no. 1, pp. 103-113, 1995.

[5] J. Shi and M. Yao, "On a singular nonlinear semilinear elliptic problem," Proceedings of the Royal Society of Edinburgh, vol. 128, no. 6, pp. 1389-1401, 1998.

[6] Z. Zhang and J. Yu, "On a singular nonlinear Dirichlet problem with a convection term," SIAM Journal on Mathematical Analysis, vol. 32, no. 4, pp. 916-927, 2000.

[7] A. V. Lair and A. W. Shaker, "Classical and weak solutions of a singular semilinear elliptic problem," Journal of Mathematical Analysis and Applications, vol. 211, no. 2, pp. 371-385, 1997.

[8] S. Yijing, W. Shaoping, and L. Yiming, "Combined effects of singular and superlinear nonlinearities in some singular boundary value problems," Journal of Differential Equations, vol. 176, no. 2, pp. 511-531, 2001.

[9] Y. Sun and S. Wu, "An exact estimate result for a class of singular equations with critical exponents," Journal of Functional Analysis, vol. 260, no. 5, pp. 1257-1284, 2011.

[10] X. Wang, L. Zhao, and P. Zhao, "Combined effects of singular and critical nonlinearities in elliptic problems," Nonlinear Analysis: Theory, Methods \& Applications, vol. 87, pp. 1-10, 2013. 
[11] Y. Haitao, "Multiplicity and asymptotic behavior of positive solutions for a singular semilinear elliptic problem," Journal of Differential Equations, vol. 189, no. 2, pp. 487-512, 2003.

[12] S. Yijing and L. Shujie, "Structure of ground state solutions of singular semilinear elliptic equations," Nonlinear Analysis: Theory, Methods \& Applications, vol. 55, no. 4, pp. 399-417, 2003.

[13] S. Goyal, "Fractional Hardy-Sobolev operator with signchanging and singular nonlinearity," Applicable Analysis, pp. 1-25, 2019.

[14] C. Y. Lei and J. F. Liao, "Multiple positive solutions for Kirchhoff type problems with singularity and asymptotically linear nonlinearities," Applied Mathematics Letters, vol. 94, pp. 279-285, 2019.

[15] J. Chabrowski, "On the Neumann problem with singular and superlinear nonlinearities," Communications in Applied Analysis, vol. 13, no. 3, pp. 327-339, 2009.

[16] H. Chen, X. Liu, and Y. Wei, "Existence theorem for a class of semilinear totally characteristic elliptic equations with critical cone Sobolev exponents," Annals of Global Analysis and Geometry, vol. 39, no. 1, pp. 27-43, 2011.

[17] D. Cao and P. Han, "Solutions for semilinear elliptic equations with critical exponents and Hardy potential," Journal of Differential Equations, vol. 205, no. 2, pp. 521-537, 2004.

[18] D. Cao, X. He, and S. Peng, "Positive solutions for some singular critical growth nonlinear elliptic equations," Nonlinear Analysis: Theory, Methods \& Applications, vol. 60, no. 3, pp. 589-609, 2005.

[19] D. Cao and S. Peng, "A note on the sign-changing solutions to elliptic problems with critical Sobolev and Hardy terms," Journal of Differential Equations, vol. 193, no. 2, pp. 424-434, 2003.

[20] J. Q. Chen and E. M. Rocha, "Positive solutions for elliptic problems with critical nonlinearity and combined singularity," Mathematica Bohemica, vol. 135, no. 4, pp. 413-422, 2010.

[21] Y. Chen and X. Tang, "Nehari-type ground state solutions for Schrödinger equations with Hardy potential and critical nonlinearities," Complex Variables and Elliptic Equations, pp. 1-21, 2019.

[22] L. Ding and C. L. Tang, "Existence and multiplicity of positive solutions for a class of semilinear elliptic equations involving Hardy term and Hardy-Sobolev critical exponents," Journal of Mathematical Analysis and Applications, vol. 339, no. 2, pp. 1073-1083, 2008.

[23] Y. Y. Shang and C. L. Tang, "Positive solutions for Neumann elliptic problems involving critical Hardy-Sobolev exponent with boundary singularities," Nonlinear Analysis, vol. 70, no. 3, pp. 1302-1320, 2009.

[24] N. Ghoussoub and X. S. Kang, "Hardy-Sobolev critical elliptic equations with boundary singularities," Annales de l'Institut Henri Poincare (C) Non Linear Analysis, vol. 21, no. 6, pp. 767-793, 2004.

[25] D. Kang and S. Peng, "Solutions for semilinear elliptic problems with critical Sobolev-Hardy exponents and Hardy potential," Applied Mathematics Letters, vol. 18, no. 10, pp. 1094-1100, 2005.

[26] Y. Lan and C. Tang, "Perturbation methods in semilinear elliptic problems involving critical hardy- sobolev exponent," Acta Mathematica Scientia, vol. 34, no. 3, pp. 703-712, 2014.

[27] J. Chen, "On a semilinear elliptic equation with singular term and Hardy-Sobolev critical growth," Mathematische Nachrichten, vol. 280, no. 8, pp. 838-850, 2007.
[28] J. P. Garcia Azorero and I. Peral Alonso, "Hardy inequalities and some critical elliptic and parabolic problems," Journal of Differential Equations, vol. 144, no. 2, pp. 441-476, 1998.

[29] J. Chen, "Multiple positive solutions for a class of nonlinear elliptic equations," Journal of Mathematical Analysis and Applications, vol. 295, no. 2, pp. 341-354, 2004.

[30] J. Chen and E. M. Rocha, "Four solutions of an inhomogeneous elliptic equation with critical exponent and singular term," Nonlinear Analysis: Theory, Methods \& Applications, vol. 71, no. 10, pp. 4739-4750, 2009.

[31] N. Hirano, C. Saccon, and N. Shioji, "Existence of multiple positive solutions for singular elliptic problems with a concave and convex nonlinearities," Advances in Differential Equations, vol. 9, no. 1-2, pp. 197-220, 2004.

[32] G. Tarantello, "Multiplicity results for an inhomogeneous Neumann problem with critical exponent," manuscripta mathematica, vol. 81, no. 1, pp. 57-78, 1993.

[33] M. Willem, Minimax Theorems, Birkhauser, Boston, 1996. 